\title{
New lichenized and lichenicolous fungi for the Crimean peninsula (Ukraine)
}

\author{
ALEXANDER KHODOSOVTSEV \\ JAN VONDRÁK \\ JAROSLAV ŠOUN
}

Khodosovtsev A., VONDRÁK J., ŠOUn J. 2007: New lichenized and lichenicolous fungi for the Crimean peninsula (Ukraine). Chornomors'k. bot. z., vol. 3, N2: 109-118.

Data on 25 species of lichenized and lichenicolous fungi new to the Crimean Peninsula, collected during Czech and Ukrainian-Czech lichenological excursions in June 2006 and May 2007 respectively, are provided. Among them, Arthonia lecanorina (Almq.) R. Sant., A. nideri (J. Steiner) Clauzade, Diederich \& Cl. Roux, A. punctella Nyl., Caloplaca furax Egea et Llimona, C. soralifera Vondrák \& Hrouzek, C. veneris Cl. Roux \& Nav.-Ros., Carbonea assimilis (Körber) Hafelner \& Hertel, Catillaria aff. scotinodes (Nyl.) Coppins, Endococcus macrosporus (Arnold.) Nyl., E. ramalinarius (Lind.) D. Hawksw., Fuscidea gothoburgensis (H. Magn.) V. Wirth \& Vězda, Lichinella cribellifera (Nyl) P.P. Moreno \& Egea, Milospium graphideorum (Nyl.) D. Hawksw., Phaeospora lecanorae Eitner, Placidium pilosellum (Breuss) Breuss, Verrucaria biatorinaria Zehetl, and $V$. poeltiana Clauzade \& Cl. Roux are new to Ukraine. Caloplaca irrubescens (Nyl. ex Arnold) Zahlbr., Lecanora rouxii S. Ekman \& Tønsberg, Lichenothelia scopularia (Nyl.) D. Hawksw., Melanelia hepatizon (Ach.) A. Thell, Peltigera collina (Ach.) Schrad., Staurothele areolata (Ach.) Lettau, Stigmidium rouxianum Calatayud \& Triebel and Zwackhiomyces coepulonus (Norm.) Grube \& R. Sant. are new to Crimea.

Key words: Arthonia, biodiversity, Caloplaca, Crimea, lichenized fungi, lichenicolous fungi, Ukraine

ХОДОСОВЦЕВ О., ВОНДРАК Я., ШОУН Я. 2007: Нові для Кримського півострова (Україна) ліхенізовані та ліхенофільні гриби. Чорноморськ. бот. ж., vol. 3, N2: 109-118.

Вперше для Кримського півострова наводиться 25 видів ліхенізованих та ліхенофільних грибів, що були зібрані учасниками чеської (червень 2006 року) та міжнародної українсько-чеської експедицій (травень 2007 року). Серед них, Arthonia lecanorina (Almq.) R. Sant., A. nideri (J. Steiner) Clauzade, Diederich \& Cl. Roux, A. punctella Nyl., Caloplaca furax Egea et Llimona, C. soralifera Vondrák \& Hrouzek, $C$. veneris Cl. Roux \& Nav.-Ros., Carbonea assimilis (Körber) Hafelner \& Hertel, Catillaria aff. scotinodes (Nyl.) Coppins, Endococcus macrosporus (Arnold.) Nyl., E. ramalinarius (Lind.) D. Hawksw., Fuscidea gothoburgensis (H. Magn.) V. Wirth \& Vězda, Lichinella cribellifera ( $\mathrm{Nyl}$ ) P.P. Moreno \& Egea, Milospium graphideorum (Nyl.) D. Hawksw., Phaeospora lecanorae Eitner, Placidium pilosellum (Breuss) Breuss, Verrucaria biatorinaria Zehetl та $V$. poeltiana Clauzade \& $\mathrm{Cl}$. Roux виявились новими для України, а Caloplaca irrubescens (Nyl. ex Arnold) Zahlbr., Lecanora rouxii S. Ekman \& Tønsberg, Lichenothelia scopularia (Nyl.) D. Hawksw., Melanelia hepatizon (Ach.) A. Thell, Peltigera collina (Ach.) Schrad., Staurothele areolata (Ach.) Lettau, Stigmidium rouxianum Calatayud \& Triebel and Zwackhiomyces coepulonus (Norm.) Grube \& R. Sant. - новими для Криму.

Ключові слова: Arthonia, різноманіття, Caloplaca, Крим, ліхенізовані гриби, ліхенофільні гриби, Україна

(C) A. Khodosovtsev, J. Vondrák, J. Šoun Chornomors'k. bot. z., vol. 3, N2: 109-118. 


\section{Introduction}

The Crimean landscapes are rich in biodiversity, particularly in respect of lichenized and lichenicolous fungi. Investigations in lichen biodiversity of the Crimea are as yet incomplete, but more than 350 species new for the Peninsula have been found there during the past 10 years [Coppins et al., 2001; KHODOSOVTSEV, 1998; ХодОСОВЦЕВ, 1997, 1999, 2000, 2001, 2002А,Б,В,Г,Д, 2003А,Б,В, 2004, 2005 А,Б,В, 2006; ХОДОСОВЦЕВ, БОГДАН, 2005; ХОДОСОВЦЕВ, ПОПОВ, 2003; ХОДОСОВЦЕВ, РЕДЧЕНКО, 2002, etc.]. Caloplaca albopustulata, C. geleverjae, C. karadagensis, and Lichenostigma svandae were described from this territory at the beginning of this century [KHODOSOVTSEV, KONDRATYUK, KÄRNEFELT, 2002, 2003; VONDRÁK, ŠOUN, 2007]. Currently, 950 species of lichenized and lichenicolous fungi are know to occur in Crimea (Khodosovtsev, unpublished data) and by this paper, a further 25 species are added to its flora.

\section{Materials and Methods}

The lichenized and lichenicolous fungi were collected in the Alushta, Feodosia and Yalta regions and the Bahchisaraysky, Leninsky and Sudaksky districts of the Crimea Autonomic Republic during a Czech lichenological excursion (J. Vondrák, J. Šoun) in June 2006 and a Ukrainian-Czech excursion (A. Khodosovtsev, J. Vondrák, J. Šoun, Yu. Khodosovtseva) in May 2007. The specimens are deposited in the herbaria of the Kherson State University (KHER) and the Faculty of Science at the University of South Bohemia (CBFS). Lichenicolous fungi are indicated by an asterisk before their names in the list below.

\section{List of taxa}

*Arthonia lecanorina (Almq.) R. Sant. in R. Sant. et al. (2004) Lichen-forming and lichenicolous fungi of Fennoscandia: 24.

?Syn. Arthonia galactinaria Leight.

Ref: ClauZade, Diederich, RouX, 1989; KoCOURKOVÁ, 2000.

Distribution in Ukraine: Crimea AR, Sevastopol, ruins of Greek town Chersones, alt. c. $10 \mathrm{~m}$, lichenicolous in apothecia of Lecanora albescens, 12.06.2006, Vondrák (CBFS JV5181).

The species is similar to Arthonia molendoi, but it is specifically lichenicolous on apothecia of Lecanora dispersa s.l. It is probably widespread in the Palearctic. New to Ukraine.

*Arthonia nideri (J. Steiner) Clauzade, Diederich \& Cl. Roux, nom. in ed.

Ref: Clauzade, Diederich, RouX, 1989.

Distribution in Ukraine: Crimea AR, Feodosia, small hills near road from Yuzhnoye to Ordzhonikidze, alt. c. $70 \mathrm{~m}, 44^{\circ} 59^{\prime} 05.97^{\prime \prime} \mathrm{N}, 35^{\circ} 18^{\prime} 15.10^{\prime \prime} \mathrm{E}$, on calcareous sandstone, lichenicolous on remains of Caloplaca variabilis s.1., 25.05.2007, Vondrák (CBFS JV6039); Karadag, close to Kurortnoye village, alt. c. $50 \mathrm{~m}, 44^{\circ} 54^{\prime} 57.85^{\prime \prime} \mathrm{N}, 35^{\circ} 12^{\prime} 15.56^{\prime \prime} \mathrm{E}$, on limestone rock, lichenicolous on Caloplaca albopruinosa, 23.05.2007, Vondrák (CBFS JV5314).

The species specifically affects members of Caloplaca subg. Pyrenodesmia. Its morphology is similar to Arthonia molendoi and its distribution is insufficiently known. New to Ukraine.

*Arthonia punctella Nyl. in Carroll, Nat. Hist. Rev. 6: 533 (1859).

Ref: Clauzade, Diederich, RouX, 1989.

Distribution in Ukraine: Crimea AR, Feodosia, small hills near road from Yuzhnoye to Ordzhonikidze, alt. c. $70 \mathrm{~m}, 44^{\circ} 59^{\prime} 05.97 " \mathrm{~N}, 35^{\circ} 18^{\prime} 15.10^{\prime \prime} \mathrm{E}$, on calcareous sandstone, lichenicolous on Aspicilia calcarea, 25.05.2007, Vondrák (CBFS).

The species affects calcicolous crustose lichens and is characterized by its dark brown hypothecium in the lower part, and brown ascospores when over-mature. Its distribution is insufficiently known. New to Ukraine. 
Caloplaca furax Egea \& Llimona, Collectanea Botanica, 14: 266 (1983).

Ref: EGEA, LLIMONA, 1983.

Distribution in Ukraine: Crimea AR, Alushta region, Cape Plaka, on Aspicilia, on vertical S surface of diorite, Vondrák, Khodosovtsev, Šoun 28.05.2007 (CBFS JV6040, KHER).

One of the authors (J. Vondrák) has checked the isotype material of Caloplaca furax [VONDRÁK, SLAVÍKOVÁ-BAYEROVÁ, 2006] and considered it a good species which differs from $C$. spalatensis, C. pellodella and C. xerica. The species is characterized by its parasitic grow on Aspicilia, grey uneven, wavy to knobby, squamulate to areolate thallus with irregular short marginal lobes, c. 0,4-0,8 mm long, without vegetative diaspores, with presence of Sedifolia-grey pigment in the cortex, zeorine apothecia with grey outer margin and orangebrownish apothecial disk. C. pellodella is a similar species, which differs by its even surfaces of glossy lead-grey squamules, blackish amphithecial ring and usually non-lichenicolous growth. The similar C. xerica, occasionally lichenicolous on Aspicilia, differs in its grey to dark grey pustules or lobules as vegetative diaspores on the surface of areoles. The calcicolous $C$. spalatensis has a flat grey areolate thallus. C. furax has been reported from Spain [EgEA, Llimona, 1983], Italy [Nimis, MARTEllos, 2003] and Bulgaria [VondráK, SLAVÍKOVÁ-BAYEROVÁ, 2006]. New to Ukraine.

Caloplaca irrubescens (Nyl. ex Arnold) Zahlbr., Verh. zool.-bot. Ges. Wien, 48: 365 (1898).

Syn. Caloplaca subsoluta (Nyl.) Zahlbr.

Ref: WETMORE, 2003.

Distribution in Crimea: Alushta region, Botanical Reserve 'Kanakskaya Balka', on schist, 27.05.2007, Vondrák, Šoun, Khodosovtsev (CBFS JV6024, KHER).

C. irrubescens has a thin, flat, areolate to subsquamulose yellow to orange thallus, orange biatorine apothecia, 0,1-0,6 $\mathrm{mm}$ in diameter with paraplectenchymatous layer under the hypothecium. The latter feature is a main character of this species against similar species. In Ukraine, it was collected from one locality in the Carpathian Mts by Nádvorník [ОКСНЕP, КОНдРАТюК, 1993A]. New to the Crimean Peninsula.

Caloplaca soralifera Vondrák \& Hrouzek, Graphis Scripta 18: 8 (2006).

Ref: VONDRÁK, HROUZEK, 2006.

Distribution in Ukraine: Crimea AR, Alushta region, Mt. Yuzhnaya Demerdji, Dolina Privideniy, on siliceous boulder, 28.05.2007, Vondrák (CBFS JV6009, KHER); Botanical Reserve 'Kanakskaya Balka', on schist, 27.05.2007, Vondrák, Khodosovtsev, Šoun (KHER); Khersonska oblast, Kakhovsky district, Kakhovskiy kanal water channel, on concrete, 15.11.2006, Khodosovtsev (KHER); Khmelnitska oblast, Kamenets-Podolsk, on limestone boulder in townwall, 6.06.2006, Vondrák (CBFS JV4594, 4595); Mykolayivska oblast, Pervomaysky district, pasture near Lyushnyubate village, alt. c. $80 \mathrm{~m}$, bank of River Pivdenny Bug, $48^{\circ} 10^{\prime} 15.30^{\prime \prime} \mathrm{N}$, 030 26'55.17"E, on granite boulder in pasture, 06.06.2006, Vondrák (CBFS JV5171, 4614).

C. soralifera is a recently described lichenized fungus [VONDRÁK, HROUZEK, 2006] with dark grey to violet-grey marginal soralia on areoles or squamules and zeorine apothecia. It is distinguished from C. xerica, C. furax and C. geleverjae by its sorediate thallus and from $C$. chlorina and $C$. virescens by its zeorine apothecia. C. soralifera is known from Austria, Bulgaria, Czech Republic, Germany, Slovakia and Romania [VONDRÁK, HrouZEK, 2006]. New to Ukraine.

Caloplaca veneris Cl. Roux \& Nav.-Ros., Bull. Soc. Linn. Provence, 43: 100 (1992).

Ref: ROUX, NAVARRO-ROSINES, 1992.

Distribution in Ukraine: Crimea AR, Sudaksky district, Cape Meganom, on vertical face of conglomerate exposed to sea, alt. c. 1 m, 26.05.2007, Khodosovtsev, Vondrák, Šoun (KHER).

$C$. veneris has an indistinct, endolithic to areolate grey thallus. Apothecia biatorine, orange, $(0,1-) 0,2-0,5(-0,8) \mathrm{mm}$ diam., parathecium prosoplectenchymatous, amphithecium 
reduced. Paraphyses 1,5-2 $\mu \mathrm{m}$ thick with swollen apical cells up to 4-6 $\mu \mathrm{m}$ in diameter. Asci 8-spored, 39-45 x 13-20 $\mu \mathrm{m}$, ascospores (8-)9-12(-13) $\times$ 5-6(-7) $\mu \mathrm{m}$, septa (2-)3-4(-5) $\mu \mathrm{m}$. It grows on calcareous rocks in the littoral zone. C. aquensis has a similar ecology and may be related to $C$. veneris, but differs in its larger apothecia 0,3-1,2 $\mathrm{mm}$ in diameter and larger ascospores 13-18 $\times$ 6-8 $\mu \mathrm{m}$, with somewhat thinner septa, 2,5-4 $\mu \mathrm{m}$ thick. C. navasiana, another similar species distributed in the Crimean Peninsula, possesses thicker septa, $(3,5-) 4,5-6,0(-9,0) \mu \mathrm{m}$, different shaped ascospores and lighter, yellow-orange apothecia. $C$. veneris is known from Cyprus, Greece [ROUX, NAVARRO-RosinES, 1992] and Italy [NIMIS, MARTELLOS, 2003]. New to Ukraine.

*Carbonea assimilis (Körber) Hafellner \& Hertel, in V. Wirth (1987) Flechten BadenWürttembergs: 511.

Ref: АНДРЕЕВ, 2003.

Distribution in Ukraine: Crimea AR, Alushta region, Botanical Reserve 'Kanakskaya Balka', on Diploschistes actinostomus, on schist, 27.05.2007, Khodosovtsev, Vondrák, Šoun (KHER).

C. assimilis has a small brown thallus up to 3-5 mm wide, black apothecia with concave disk and flexuose distinct margin resembling Rimularia insularis. However, Carbonea assimilis differs by its emerald-green epihymenium and Lecanora-type asci. Nonlichenicolous $C$. vorticosa has dirty-white to grey thallus and brown-black hypothecium. The third Ukrainian species, C. vitellinaria is a lichenicolous fungus on thallus of Candelariella vitellina and its apothecial structure is similar to C. vorticosa. Carbonea assimilis is known from Europe, Asia (China) and North America [АндРЕЕв, 2003]. New to Ukraine.

Catillaria aff. scotinodes (Nyl.) Coppins, Lichenologist 21: 223 (1989).

Ref: COPPINS, 1992.

Distribution in Ukraine: Crimea AR, Sudak region, Sudak, coastal rocks at W part of

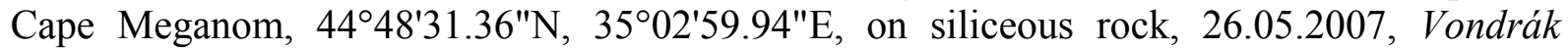
(CBFS JV5924).

The specimen from Crimea agrees with the description of $C$. scotinodes in CoPPINS [1992] in most characters, but its epihymenium is brownish (K-, N-).

*Endococcus macrosporus (Arnold) Nyl., Bull. Soc. Bot. France 25: 504 (1878).

Ref: SERUSIAUX et al., 1999.

Distribution in Ukraine: Crimea AR, Alushta region, Luchyste village, Mt. Yuzhnaya Demerdji, Dolina Privideniy, on Rhizocarpon geographicum subsp. lindsayanum, on conglomerate, 28.05.2007, Khodosovtsev, Vondrák, Šoun (KHER).

According to the narrow species concept of SERUSIAUX et al. [1999], three different species of Endococcus are lichenicolous on species of Rhizocarpon. Among them, Endococcus macrosporus is characterized by its perithecia, 130-220 $\mu \mathrm{m}$ diam., immersed in host areoles and its large ascospores, 16,5-19,5 × 5,5-7 $\mu \mathrm{m}$. As yet, known only from Luxembourg [SERUSIAUX et al., 1999]. New to Ukraine.

*Endococcus ramalinarius (Lind.) D. Hawksw., Bot. Notiser 132: 287 (1979).

Ref: HAWKSWORTH, 1979a.

Distribution in Ukraine: Crimea AR, Bakhchisaraysky district, pasture c. $500 \mathrm{~m} \mathrm{~W}$ of Mashino village, $200 \mathrm{~m}, 44^{\circ} 41^{\prime} 50.95^{\prime \prime} \mathrm{N}, 033^{\circ} 01^{\prime} 46.16^{\prime \prime} \mathrm{E}$, on Ramalina canariensis on bark of Quercus pubescens, 10.06.2006, Vondrák (CBFS JV5240).

The species was described from New Zealand as lichenicolous on Ramalina leiodea, but in Spain it was recorded on R. farinacea [MARTínEZ, 2002]. New to Ukraine. 
Fuscidea gothoburgensis (H. Magn.) V. Wirth \& Vězda, Beitrage Naturk. Forsch. Sudw.Deutschl. 31: 92 (1972).

Ref: OBERHOLLENZER, WIRTH, 1984; MAKAPOBA, 2004.

Distribution in Ukraine: Crimea AR, Alushta region, Botanical Reserve 'Kanakskaya Balka', on schist, 27.05.2007, Khodosovtsev, Vondrák, Šoun (KHER).

$F$. gothoburgensis has a black, fine prothallus with disperse convex greyish areoles, rare green-greyish soralia $(0,2-0,4 \mathrm{~mm}$ diam.) surrounded by thin thallus margin and negative chemical tests are its main characters. Widespread in Europe [MAKAPOBA, 2004]. New to Ukraine (but lacking apothecia).

Lecanora rouxii S. Ekman \& Tønsberg, Mycol. Res. 108: 512 (2004).

Syn. Lepraria flavescens Clauzade \& Roux

Ref: BARUFFo et al. 2006; ClauZADE, RouX, 1977.

Distribution in Crimea: Bakhchisaray, limestone cliff above town, alt. $300 \mathrm{~m}$, $44^{\circ} 45^{\prime} 04.68^{\prime \prime} \mathrm{N}, 033^{\circ} 53^{\prime} 06.88^{\prime \prime} \mathrm{E}$, on well-lit limestone rock under overhang, 9.06.2006, Vondrák, Šoun (CBFS JV4588, sub Lepraria flavescens).

Recent phylogenetic analyses support the placement of Lepraria flavescens in the Lecanora rupicola group [GRUBE, BALOCH, ARUP, 2004] and the "nomen novum" L. rouxii was introduced. It is an easily identified leprarioid species with lobate, $\mathrm{C}+$ orange thallus (atranorin, sordidon and flavescin). It is known from Europe [e.g. ClaUZADE, RouX, 1977; SERUSIAUX et al., 1999; WIRTH, 1995; BIELCZYK et al., 2005] and fertile specimens were recently found in Luxembourg [KUKWA, DIEDERICH, 2007]. New to Crimea.

*Lichenothelia scopularia (Nyl.) D. Hawksw., Lichenologist, 13: 147 (1981).

Ref: HAWKSWORTH, 1981; HENSSEN, 1987.

Distribution in Crimea: Alushta region, Botanical Reserve 'Kanakskaya Balka', on schist, 27.05.2007, Khodosovtsev, Vondrák, Šoun (KHER).

This microfungus has a blackish cracked non-lichenized thallus developing on naked siliceous rocks, with immersed perithecia c. $2 \mathrm{~mm}$ wide and ascospores often up to 4-celled, about 16-22 $\times 8-11 \mu \mathrm{m}$. L. convexa differs in its smaller dot-like 'thallus' and ascospores, 11$13(-15) \times 5,5-7,5 \mu \mathrm{m}$. L. scopularia is known in Europe and North America [NIMIS, 1993]; it has recently been found in Ukraine from Polissya [ФЕДОРЕНКО, НАДєІНА, КОНДРАТЮК, 2007]. New to Crimea.

Lichinella cribellifera P.P. Moreno \& Egea, Cryptogamie, Bryol. Lichénol., 13: 3: 243 (1992).

Ref: MORENO, EGEA, 1992.

Distribution in Ukraine: Crimea AR, Sudaksky district, above Vesele village, alt. c. $200 \mathrm{~m}, 4^{\circ} 50^{\prime} 57.04^{\prime \prime} \mathrm{N}, 034^{\circ} 52^{\prime} 05.87^{\prime \prime} \mathrm{E}$, on lime-rich rock, with some other cyanolichens, 13.06.2006, Vondrák (CBFS JV5260, 5286); Karadag Mts, Mt Svyataya, alt. 320 m,

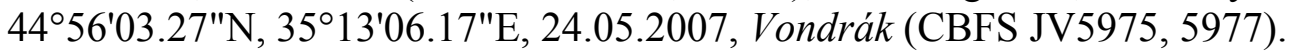

The species is similar to Lichinella nigritella, but differs in its thinner and warped lobes. New to Ukraine.

Melanelia hepatizon (Ach.) A. Thell, Nova Hedwigia 60: 419 (1995).

Ref: Rico et al., 2005.

Distribution in Crimea: Alushta region, Luchyste village, Mt. Yuzhnaya Demerdji, Dolina Privideniy, on conglomerate, 28.05.2007, Khodosovtsev, Vondrák, Šoun (KHER).

Reports of this lichen from the Carpathian Mts, mainly in 1920-1940, were made by M. Servít, J. Nádvorník, J. Suza, T. Sulma and J. Hruby [ОКСНЕР, КОНДРАТюК, 1993б]; it has also been collected M. hepatizon from Mt. Pip Ivan (Zakarpatska oblast) [МАКАРЕВИЧ и др., 1982]. New for Crimea. 
*Milospium graphideorum (Nyl.) D. Hawksw., Trans. Br. mycol. Soc. 65: 228 (1975)

Ref: HAWKSWORTH, 1975, 1979b.

Distribition in Ukraine: Crimea AR, Bakhchisaraysky district, Mashine village, on base of limestone cliff $500 \mathrm{~m} \mathrm{NW}$ of village, alt. c. $350 \mathrm{~m}, 44^{\circ} 42^{\prime} 03.87^{\prime \prime} \mathrm{N}, 033^{\circ} 54^{\prime} 26.49^{\prime \prime} \mathrm{E}$, lichenicolous on Dirina stenhammari, 10.06.2006, Vondrák (CBFS JV4613); Yalta region, Nature Reserve 'Cape Martian', on Dirina stenhammari and Lecanographa grumulosa growing on vertical surfaces of limestone rock, alt. $50 \mathrm{~m}, 44^{\circ} 30^{\prime} 26.3^{\prime \prime} \mathrm{N}, 034^{\circ} 14^{\prime} 50.8^{\prime \prime} \mathrm{E}$, 29.05.2007, Khodosovtsev, Vondrák, Šoun (CBFS JV5020, KHER).

This lichenicolous hyphomycete is characterized by its lobate, brown conidia, with 2-6 mostly incomplete septa and unevenly thickened walls, $8-12 \times 8-10 \mu \mathrm{m}$ in size, which are often aggregated into black sporodochia on a host thallus. It was previously known from Spain and Portugal [HAWKSWORTH, 1975; VAN DEN BOOM, ETAYO, 2000; VAN DEN BOOM, 1999]. New to Ukraine.

Peltigera collina (Ach.) Schrad., J. Bot., 1: 78 (1803).

Ref: VITIKAINEN, 1994.

Distribution in Crimea: Alushta region, Alupka, Ai-Petrinska yaila, c. $1 \mathrm{~km} \mathrm{~S}$ of Mt. Bedene-Kyr, alt. c. $1000 \mathrm{~m}, 4^{\circ} 28^{\prime} 28.25^{\prime \prime} \mathrm{N}, 033^{\circ} 01^{\prime} 46.16^{\prime \prime} \mathrm{E}$, on bark of Carpinus betulus, 11.06.2006, Vondrák, Šoun (CBFS JV5051).

This species is reported from the Carpathian Mts [МАКАРЕВИч и др., 1982; KONDRATYUK et al., 2003] and plain part of Ukraine [KONDRATYUK et al., 1998]. New to the Crimean Peninsula.

*Phaeospora lecanorae Eitner, Jahresber. Schles. Ges. Vaterl. Cult., 78 ('1900’), 2. Abt., b: 26 (1901).

Ref: SERUSIAUX et al., 1999.

Distribution in Ukraine: Crimea AR, Alushta region, Botanical Reserve 'Kanakskaya Balka', on Lecanora dispersa, on schist, Khodosovtsev, 27.05.06 (KHER).

This lichenicolous fungus on Lecanora dispersa agg. has perithecioid ascomata 120$220 \mu \mathrm{m}$ in diameter and brown, 3-septate ascospores 12,5-16 $\times 5,5-6 \mu \mathrm{m}$ in size [SERUSIAUX et al., 1999]. The similar P. parasitica grows on Rhizocarpon and has larger ascospores (18$23 \times 8-11,5 \mu \mathrm{m})$. P. lecanorae was described from Silesia [EITNER, 1901]; it was recently recorded from Luxembourg and France [SERUSIAUX et al., 1999]. New to Ukraine.

Placidium pilosellum (Breuss) Breuss, Ann. Naturhist. Mus. Wien., 98 B Suppl.: 39 (1996).

Ref: BREUSS, 1990.

Distribution in Ukraine: Crimea AR, Feodosiya region, WSW of Koktebel village, alt. $200 \mathrm{~m}, 44^{\circ} 57^{\prime} 31.12^{\prime \prime} \mathrm{N}, 035^{\circ} 12^{\prime} 22.93^{\prime \prime} \mathrm{E}$, limestone hill, on calcareous soil with Collema tenax and Fulgensia subbracteata, 13.06.2006, Vondrák, Šoun (CBFS JV5041).

Placidium pilosellum is distinguished from $P$. squamulosum, a widespread species in Ukraine, by its colourless hairs on the margins of squamules and by marginal picnidia. It is known from Europe and Australia [BREUSS, 1996]. New to Ukraine.

Staurothele areolata (Ach.) Lettau, Hedwigia, 52: 84 (1912).

Ref: FOUCARD, 2001.

Distribution in Crimea: Alushta region, Luchyste village, Mt. Yuzhnaya Demerdji, Dolina Privideniy, on conglomerate, 28.05.2007, Khodosovtsev, Vondrák, Šoun (KHER).

This species is known from few sites in the Carpathian Mts [МАКАРЕВИЧ и др., 1992] and Ukrainian plain [KONDRATYUK et al., 1998]. New for Crimean Peninsula.

*Stigmidium rouxianum Calatayud \& Triebel, Lichenologist, 35: 109 (2003). 
Ref: CALATAYUD, TRIEBEL, 2003.

Distribution in Crimea: Bakhchisaraysky district, pasture c. $500 \mathrm{~m} \mathrm{~W}$ of Mashino village, $350 \mathrm{~m}, 4^{\circ} 42^{\prime} 03.87^{\prime \prime} \mathrm{N}, 033^{\circ} 54^{\prime} 26.49^{\prime \prime} \mathrm{E}$, on thallus of Acarospora cervina, 10.06.2006, Vondrák, Šoun (CBFS JV4583); Alushta region, Alupka, Ai-Petrins 'ka yaila, c.1 $\mathrm{km}$ S from Mt. Bedene-Kyr, alt. c. $1100 \mathrm{~m}, 44^{\circ} 28^{\prime} 28.25^{\prime \prime} \mathrm{N}, 034^{\circ} 01^{\prime} 46.16 " \mathrm{E}$, on thallus of Acarospora cervina, 11.06.2006, Vondrák, Šoun (CBFS JV4589); Luchyste village, Mt Yuzhnaya Demerdji, Dolina Privideniy, on thallus of Acarospora cervina, on conglomerate, 28.05.2007, Khodosovtsev, Vondrák, Šoun (KHER).

As well as being restricted to Acarospora cervina, Stigmidium rouxianum is characterized by its negative reaction with Lugol's solution and ascospore size, (14-)14.5-18(21) $\times(5-) 6-7(-8) \mu \mathrm{m}$ [CALATAYUD, TRIEBEL, 2003]. It is known from Spain, France, Italy, Switzerland and western Ukraine [CALATAYUd, Triebel, 2003; BIELCZYK et al., 2005]. New to Crimea.

Verrucaria biatorinaria Zehetl., Nova Hedwigia, 29: 721 (1978).

Syn. Verrucula biatorinaria (Zehetl.) Nav.-Ros. \& Cl. Roux.

Ref: NAVARRO-RosinÉs et al., 2007; ZEHETLEITNER, 1978.

Distribution in Ukraine: Crimea AR, Alushta region, Cape Plaka, on diorite, lichenicolous on thallus of Caloplaca biatorina, 28.05.2007, Vondrák (CBFS JV5984); Alushta region, Luchyste village, Mt. Yuzhnaya Demerdji, Dolina Privideniy, on Caloplaca biatorina, on conglomerate, 28.05.2007, Khodosovtsev, Vondrák, Šoun (KHER); Sudaksky district, Sudak, Juniperus forest in 'Novy Svet' Nature Reserve, alt. 50 m, 4449'31.16"N, 34'54'19.02"E, lichenicolous on Caloplaca biatorina, 26.05.2007, Vondrák, Khodosovtsev (CBFS JV6003, KHER).

Verrucaria biatorinaria forms greyish areoles on Caloplaca biatorina with negative medulla reaction with Lugol's solution. It has immersed perithecia with black peridium (centre 150-200 $\mu \mathrm{m}$ ) and broadly ellipsoid ascospores, $8-14 \times 7-8 \mu \mathrm{m}$ in size. It is known from the Alps (Austria, Italy, France), Spanish Pyrenees and Kurdistan [e.g. NAVARroRosINÉS et al., 2007; Nimis, 1993]. New to Ukraine.

Verrucaria poeltiana Clauzade \& Cl. Roux, Beih. Nova Hedwigia, 79: 196 (1984).

Syn. Verruculopsis poeltiana (Clauzade \& Cl. Roux) Gueidan, Nav.-Ros. \& Cl. Roux.

Ref: ClauZADE, RouX, 1984; NAVARro-RosinÉs et al., 2007.

Distribution in Ukraine: Crimea AR, Yalta region, Nature Reserve 'Cape Martian', on limestone rock, lichenicolous on thallus of Caloplaca aurantia with Lichenostigma sp., alt. 50 m, 4430'26.3"N, 034²14'50.8"E, 29.05.2007, Khodosovtsev, Vondrák, Šoun (CBFS JV5430, KHER).

This lichenicolous lichen, known only from Caloplaca aurantia, is dubiously distinguished from some other grey-thallus species described from different Caloplaca and Xanthoria species, but NAVARRO-ROSINÉs et al. [2007] consider it as a well-defined taxon. Known from France, Italy and Spain [NAVARRO-ROSINÉS et al., 2007]. New to Ukraine.

*Zwackhiomyces coepulonus (Norman) Grube \& R. Sant., in Grube \& Hafellner, Nova Hedwigia, 51: 310 (1990).

Ref: GRUBE, HAFELLNER, 1990.

Distribution in Ukraine: Crimea AR, Sudaksky district, Cape Meganom, on Caloplaca maritima growing on vertical face of conglomerate rock, alt. c. $1 \mathrm{~m}, 26.05 .2007$, Khodosovtsev, Vondrák, Śoun (KHER). Mikolayivska oblast, Pervomaiskky district, pasture near Lyushnyuvate village, alt. c. $80 \mathrm{~m}, 48^{\circ} 10^{\prime} 15.30^{\prime \prime} \mathrm{N}, 030^{\circ} 26^{\prime} 55.17^{\prime \prime} \mathrm{E}$, in apothecia and thallus of Caloplaca crenulatella growing on nutrient-rich granite rock, 6.06.2006, Vondrák (CBFS JV5155).

This lichenicolous fungus was once reported from Ukraine, natural reserve Medobory [КондРАТЮК, КолОмІєць, 1997]. New for the Crimean Peninsula. 


\section{Acknowledgements}

We are grateful to the Director of the "Cape Martian" Nature Reserve of the Nikitsky Botanical Garden - National Science Centre (Yalta, Crimea AR), to Prof. Dr I. Maslov and his wife Dr I. Sarkina for hospitality and substantial help during fieldwork. The financial support of the Grant of President of Ukraine № GP/F13/0196 (A. Khodosovtsev) and the Grant of Academy of Sciences of the Czech Republic KJB 601410701 (J. Vondrák \& J. Šoun) are gratefully acknowledged. Last but not least, Prof. M. R. D. Seaward kindly provided useful criticisms and linguistic corrections.

\section{Literature}

АНДРЕЕВ М.П. Сем. Lecanoraceae. - В кн. Андреев М.П., Бредкина Л.И., Голубкова Н.С. и др. Определитель лишайников России. Вып. 8. Бацидиевые, Катиляриевые, Леканоровые, Мегалариевые, Микобилимбиевые, Ризокарповые, Трапелиевые. - Спб.: Наука, 2003. - С.111-184.

КОНдРАТЮК С.Я., КОЛОмІЦь І.В. Нові для України види лишайників та ліхенофільних грибів заповідника «Медобори» // Укр. ботан. журн. - 1997. - Т. 54, № 1. - С. 43-47.

МАКАРЕВИЧ М.Ф., НАВРОЦКАЯ И.Л. ЮДИНА И.В. АТЛас географического распространения ЛишайНиков в Украинских Карпатах. - К.: Наук. думка, 1982. - 403 с.

МАКАРОВА И.И. Сем. Fuscideaceae. - В кн. Кондратюк С.Я., Макарова И.И., Окснер А.М., Ходосовцев А.Е. Определитель лишайников России. Вып. 9. Фусцидеевые, Телосхистовые. - Спб.: Наука, 2004. - С.1036.

ОкСНЕР А. М., КОНДРАТЮК С.Я. Рід 150. Калоплака - Caloplaca Th. Fr. - В кн.: Окснер А.М. Флора лишайників України. - К.: Наук. думка, 1993а. - Т. 2, вип. 2. - С. 390-490.

ОкСНЕР А. М., КОНДРАТЮК С.Я. Рід 135. Цетрарія - Cetraria Ach. - В кн.: Окснер А.М. Флора лишайників України. - К.: Наук. думка, 1993б. - Т. 2, вип. 2. - С. 225-248.

ХодосовцЕв О.С. Новий для ліхенофлори України рід Dirina Fr. // Укр. ботан. журн. - 1997. - Т.54, №5. - C. 381383.

ХодосовЦЕВ О.С. Лишайники причорноморських степів України. - Київ: Фітосоціоцентр, 1999. - 236 с.

ХодосовЦЕв О.С. Нові для Криму та України види лишайників // Укр. ботан. журн. - 2000. - Т.57, №5. - С. 612615.

Ходосовцев О.Є. Нові для України види роду Caloplaca Th. Fr. (Teloschistaceae) // Укр. ботан. журн. - 2001. Т.58, № 4. - С. 460-465.

ХодосОвцЕВ О.С. Нові для України та Кримського півострова види лишайників з Кримських яйл // Укр. ботан. журн. - 2002а. - Т.59, №2. - С. 171-178.

ХодосовцЕв О.Є. Нові та рідкісні для України види роду Caloplaca Th. Fr. (Teloschistaceae) з півдня України // Укр. ботан. журн. - 2002б. - Т.59, № 3. - С. 321-329.

ХодосовцЕв O.Є. Absconditella Vezda (Ostropales) та Gonohymenia J. Steiner (Lichinales) - нові роди для ліхенофлори Кримського півострова // Укр. ботан. журн. - 2002в. - Т.59, № 5. - С. 612-615.

ХодосовЦЕВ О.С. Екологічні індекси лишайників кам'янистих відслонень Карабі-яйли (АР Крим, Україна) // Природничий Альманах. Серія: Біологічні науки. - Вип. 2, № 3. - Херсон, 2002г. - С. 225-239.

ХодосовцЕВ О.С. Лишайники карстовых обнажений Чатырдага (Крым) // Ботан. журн. - 2002д. - Т.87, № 1. C. $46-56$.

ХодосовцЕв О.Є. Нові для України та Криму види лишайників з силікатних відслонень // Укр. ботан. журн. 2003a. - T.60, №1. - С. 70-78.

Ходосовцев О.С. Рід Bagliettoa А. Massal. (Verrucariales Mattik ex D. Hawksw. \& O. Eriksson) Кримського півострова (Україна) // Укр. ботан. журн. - 2003б. - Т.60, №2. - С. 131-138.

ХодосовцЕВ О.С. Анотований список лишайників Карадазького природного заповідника // Вісті Біосферного заповідника "Асканія-Нова". - 2003в. - Т.5. - С. 31-43.

ХодосовцЕв O.C. Lichenothelia D.Hawksw. - новий рід для мікобіоти України // Укр. ботан. журн. - 2004. - Т.61, №6. - C. 32-34.

Ходосовцев O.Є. Placopyrenium O. Breuss - (Lichens, Verrucariaceae) новий рід для ліхенофлори Кримського півострова // Укр. ботан. журн. - 2005а. - Т.62, №1. - С. 111-114.

Ходосовцев О.С. Нові для України роди лишайників // Укр. ботан. журн. - 2005б. - Т.62, №2. - С. 170-174.

ХодосовцЕв А.Е. Род Candellariella (Candelariaceae, Lecanorales) юга Украины // Новости систематики низших растений. - Т. 39. - 2005в. - С. 233-248.

ХодосовЦЕв О.С. Нові для України види лишайників з Криму // Укр. ботан. журн. - 2006. - Т. 63, № 2. - С. 196202. 
ХодосовцЕв О.Є., БогдАн О.В. Анотований каталог лишайників Ялтинського гірсько-лісового природного заповідника // Чорномор. ботан. журн. - 2005. - Т.1, №1 . - С. 117-132.

ХодосовцЕв О.С., Попов Є.В. Leptogium imbicatum P. Jørg. та L. magnussonii Degel. \& P. Jorg. нові види для ліхенофлори України // Метода. - 2003. - Вип. “Магістр”. - С. 25-28.

ХодосовцЕв О.С., РЕдчЕНКО О.О. Анотований список лишайників заповідника "Мис Март'ян" // Укр. ботан. журн. - 2002. - Т.59, № 1. - С. 64-71.

ФЕДОРЕНКО Н.М., НАДЄІНА О.В., КОНДРАТЮК С.Я. Нові та рідкісні види ліхенофільних грибів з України // Укр. ботан. журн. - 2007. - Т. 64, № 1. - С. 47-56.

BARUFFO L., ZEDDA L., ELIX J.A., TRETIACH M. A revision of the lichen genus Lepraria s.lat. in Italy // Nova Hedwigia. 2006 - Vol. 83. - P. 387-429.

BIELCZYK U. et al. Contribution to the knowledge of lichens and lichenicolous fungi of western Ukraine // Polish Botanical Journal. - 2005. - Vol. 50, № 1. - P. 39-64

BOOM P.P.G. VAN DEN. Some lichens and lichenicolous fungi from Majorca (Spain) // Linzer boil. Beitr. - 1999. - Vol. 31, N2. - P. 785-800.

BOOM P.P.G. VAN DEN., ETAYO J. Contribution to the knowledge of lichenicolous fungi and lichens from Portugal and Spain // Öster. Z. Pilzk. - 2000. - N9. - P. 151-162.

BREuss O. Die flechtengattung Catapyrenium (Verrucariaceae) in Europa // Stapfia. - 1990. - Vol. 23. - P.1-174.

BREUSS O. Ein verfeinertes Gliederungskonzept für Catapyrenium (Lichenisierte Ascomyceten, Verrucariaceae) mit einem Schlüssel für die bisher bekannten Arten // Ann. Naturhist. Mus. Wien. - 1996. - Suppl. 98 B. - P. 35-50.

Calatayud V., TRIeBel D. Three new species of Stigmidium s.l. (lichenicolous ascomycetes) on Acarospora and Squamarina // Lichenologist. - 2003. - Vol. 35, N 2. - P. 103-116.

ClauzAdE G., Diederich P., RouX C. Nelikenigintaj fungoj likenlogaj. Ilustrita determinlibro // Bulletin de la Societe Linneenne de Provence, Numero Special - 1989. - Vol. 1. - P. 1-142.

ClauzAdE G., Roux C. Taxons nouveaux et intéressant pour le de la France // Bull. Soc. Linn. Provence. - 1977. - Vol. 30. - P. 9-36.

ClauzADE G., Roux C. Deux especes nouvelles de lichens mediterraneens: Lecanora poeltiana Clauz. et Roux sp. nov., Verrucaria poeltiana Clauz. et Roux sp. nov. // Beiheft zur Nova Hedwigia - 1984. - Vol. 79. - P. 187-201.

CoppINS B.J. Catillaria - In: O.W. Purvis et al. (eds.): The lichen flora of Great Britain and Ireland. - Natural History Museum Publications \& British Lichen Society, London. - 1992. - P. 166-171.

Coppins B., KondRatyUK S.YA., KhodosovtSeV A.Ye., Wolseley P., ZelenKo S.D. New for Crimea and Ukraine Species of the lichens // Укр. ботан. журн. - 2001. - Т. 58, №6. - С. 716-722.

EgEA J.M., Llimona X. Caloplaca furax Egea \& Llimona, un Nuevo liquen parasito sobre Aspicilia siliciolas, en la Sierra del Relumbar (Albacete, SE de Espana) // Collectanea Botanica. - 1983. - N14. - P. 265-269.

EITNER E. II. Nachtrag zur Schlesischen Flechtenflora // Jahresber. Schles. Ges. Vaterl. Cult. - 1901. - Vol. 78 ('1900'), N 2, Abt., b. - P. 5-27.

FOUCARD T. Svenska Skorplavar - Interpublishing, Stockholm, 2001.

GRUBE M. BALOCH E., ARUP. U. A phylogenetic study of the Lecanora rupicola group (Lecanoraceae, Ascomycota) // Mycol. Res. - 2004. - Vol. 108, N 5. - P. 506-514.

Grube M., HAFELlner J. Studien an flechtenbewohnenden Pilzen der ammelgattung Didimella (Ascomycetes, Dothideales) // Nova Hedwigia. - 1990. - Vol. 51. - P. 283-360.

HAwKSWORTH D.L. A revision of lichenicolous fungi accepted by Keissler in Coniothecium // Trans. Br. Mycol. Soc. 1975. - Vol. 65. - P.219-238.

HAWKSWORTH D.L. Studies in the genus Endococcus (Ascomycotina, Dothideales) // Botaniska Notiser. - 1979a. Vol. 132. - P. 283-290.

HAWKSWORTH D.L. The lichenicolous Hyphomycetes // Bulletin of the British Museum (Natural History), Botany Series - 1979b. - Vol. 6. - P. 183-300.

HAWKSWORTH D. L. Lichenothelia, a new genus for the Microthelia alterrima group // Lichenologist. - 1981. - Vol. 13, N 2. - P. 141-153.

HeNSSEN A. Lichenothelia, a genus of microfungi on rocks. - In.: Peveling E. (ed.): Progress and Problems in Lichenology in the Eighties // Bibl. Lichenol. - 1987. - Vol. 25. - P. 257-293.

KHODOSOVTSEV A.YE. New lichen species for the biota of Ukraine // Ukr. botan. jurn. - 1998. - Vol. 55, N1. - P. 88-9I.

KhODOSOVTSEV A. Ye., KondRATYUK S.YA., KäRNEFElt I. Caloplaca albopustulata, a new lichen from Crimea peninsula (Ukraine) // Graphis Scripta. - 2002. - Vol. 13, N 1. - P. 5-8.

KhodosovtSeV A.Ye., KondRATYUK S.YA., KÄRNEFELT I. Two new species of Caloplaca from Crimean peninsula // Ukr. botan. journ. - 2003. - T.60, N3. - P. 293-297.

KocourKovÁ J. Lichenicolous fungi of the Czech Republic (The first commented checklist) // Acta Mus. Nat. Pragae, Ser. B., Hist. Nat. - 2000. - Vol. 55/1999. - P. 59-169.

KONDRATYUK S.YA., KHODOSOVTSEV A.YE.. ZelenKo S.D. The second checklist of lichen forming, lichenicolous and allied fungi of Ukraine. - Kiev: Phytosociocentre, 1998. - $180 \mathrm{p}$.

KONDRATYUK S.YA., POPOVA L.P., LACKOVIČOVÁ A., PIŠUT I. A catalogue of the Eastern Carpathian Lichens. - KievBratislava: M.H. Kholodny Institute of Botany, 2003. -264 p. 
KUKWA M., DIEDERICH P. New records of leprarioid lichens from Luxembourg and France with first report of fertile Lecanora rouxii // Bull. de la Soc. des nature. luxemburg. - 2007. - Vol. 108. - P. 15-19.

MARTínEZ I. Lichenicolous fungi from the Iberian Peninsula and the Macaronesian area // Nova Hedwigia. - 2002. Vol. 74. - P. 51-67.

Moreno P.P., EgEA J.M. El genero Lichinella Nyl. En el sureste de Espana y norte de Africa // Criptogamie, Bryol. Lichenol. - 1992. - Vol. 13, N 3. - P. 237-260.

NAVArRo-Rosines P., Roux C., GueIDAN C. La genroj Verrucula kaj Verruculopsis (Verrucariaceae, Verrucariales) // Bull. Soc. Linn. Provence. - 2007. - Vol. 58. - P. 133-180.

NIMIS P. L. The Lichens of Italy. An annotated cataloque. - Monograpfie XII. - Torino, 1993. - 897 p.

NIMIS P.L., MARTELLOS S. A second checklist of the lichens of Italy with thesaurus of synonyms. - Museo Regionale di Scienze Naturali. - Aosta, 2003.

OBERHOLLENZER H., WIRTH V. Beitrage zur Revision der Flechtengattung Fuscidea // Nova Hedwigia. - 1984. - Bd 79. - S. 537-595.

RICO V.J., BOOM P.P.G. VAN DEN, BARRosA J.M. Morphology, chemistry and distribution of Melanelia sorediella (Parmeliaceae) and similar species in Iberian peninsula // Lichenologist. - 2005. - Vol. 37, N3. - P. 199-215.

Roux C., NAVARro-Rosines P. Caloplaca egeana Roux et Nav.-Ros. sp. nov. kaj Caloplaca veneris Roux et Nav.-Ros sp. nov., du novaj likenspecioj de la mediterranea marbordo // Bull. Soc. Linn. Provence. - 1992. - Vol. 43. P. 97-103.

Serusiaux E., Diederich P., Brand A.M., BoOM P.P.G.VAN DEN. New or interesting lichens and lichenicolous fungi from Belgium and Luxemburg. VIII. // Lejeunia. - 1999. - Vol. 162. - P. 1-96.

VITIKAINEN O. Taxonomic revision of Peltigera (lichenized Ascomycotina) in Europe // Acta Botanica Fennica. - 1994. - Vol. 152. - P. 1-96.

VONDRÁK J., HROUZEK P. Caloplaca soralifera, a new species from Europe // Grapis Scripta. - 2006. - Vol. 18. - P. 615.

VONDRÁK J., SLAVÍKOVÁ-BAYEROVÁ S. Contribution to the lichenized and lichenicolous fungi in Bulgaria. II, the genus Caloplaca // Mycologica Balcanica. - 2006. - N 3. - P. 61-69.

VONDRÁK J., ŠOun J. Lichenostigma svandae, a new lichenicolous fungus on Acarospora cervina // Lichenologist. 2007. - Vol. 39, N3. - P. 211-216.

WetMore C.M. The Caloplaca squamulosa group in North and Central America // The Bryologist. - 2003. - Vol. 106, N1. - P. 147-156.

WIRTH V. Die Flechten Baden-Württembergs. - Ulmer, Stuttgart, 1995. - Vol. 1-2. - 1006 p.

ZEHETLEITNER G. Über einige parasitische Arten der Flechtengattung Verrucaria // Nova Hedwigia. - 1978. - Vol. 29. P. 683-734.

Рекомендує до друку

Отримано 10.10.2007

М.Ф. Бойко

Aдреси авторів:

О.С. Ходосовиев

Херсонський державний університет, кафедра

ботаніки

вул. 40 років Жовтня, 27

Херсон, 73000

Україна

e-mail:khodosovtsev@ksu.ks.ua

Я. Вондрак, Я. Шоун

Кафедра ботаніки наукового факультету

Університету Південної Богемії

Бранішовська, 31

CZ-37005

Чеське Будейовице

Чеська Республіка

e-mail:j.vondrak@seznam.cz
Author's addresses:

A. Ye. Khodosovtsev

Kherson State University

27, 40 Rokiv Zhovtnya str.

Kherson, 73000

Ukraine

e-mail:khodosovtsev@ksu.ks.ua

J. Vondrák, J. Šoun

Department of Botany,

Faculty of Sciences,

University of South Bohemia

Branišovská, 31

CZ-37005,

České Budějovice,

Czech Republic

e-mail:j.vondrak@seznam.cz 